\title{
BMJ Open Exploratory clinical trial of combination wound therapy with a gelatin sheet and platelet-rich plasma in patients with chronic skin ulcers: study protocol
}

\author{
Naoki Morimoto, ${ }^{1}$ Natsuko Kakudo, ${ }^{1}$ Makoto Matsui, ${ }^{2}$ Tsunetaka Ogura, ${ }^{1}$ \\ Tomoya Hara, ${ }^{1}$ Kenji Suzuki, ${ }^{1}$ Masaya Yamamoto, ${ }^{2}$ Yasuhiko Tabata, ${ }^{2}$ \\ Kenji Kusumoto ${ }^{1}$
}

To cite: Morimoto N, Kakudo N, Matsui M, et al. Exploratory clinical trial of combination wound therapy with a gelatin sheet and platelet-rich plasma in patients with chronic skin ulcers: study protocol. BMJ Open 2015;5:e007733. doi:10.1136/bmjopen-2015007733

- Prepublication history for this paper is available online. To view these files please visit the journal online (http://dx.doi.org/10.1136/ bmjopen-2015-007733).

Received 20 January 2015 Revised 24 March 2015 Accepted 26 March 2015

\section{CrossMark}

${ }^{1}$ Department of Plastic and Reconstructive Surgery, Kansai Medical University, Osaka, Japan

${ }^{2}$ Department of Biomaterials Field of Tissue Engineering, Institute for Frontier Medical Sciences, Kyoto University, Kyoto, Japan

Correspondence to Dr Naoki Morimoto; morimotn@hirakata.kmu.ac.jp

\begin{abstract}
Introduction: Chronic skin ulcers, such as diabetic ulcers, venous leg ulcers and pressure ulcers, are intractable and increasing in prevalence, representing a costly problem in healthcare. We developed a combination therapy with a gelatin sheet, capable of providing sustained release of platelet-rich plasma (PRP). The objective of this study is to investigate the safety and efficacy of autologous PRP covered with a hydrocolloid dressing and PRP covered with a gelatin sheet in the treatment of chronic skin ulcers.
\end{abstract}

Methods and analysis: Thirty patients with chronic skin ulcers who have not healed with conventional therapy for at least 1 month are being recruited. The patients will receive PRP after debridement, and the wounds will be covered with a hydrocolloid dressing or gelatin sheet. The efficacy will be evaluated according to the time from the beginning of PRP application to secondary healing or the day on which wound closure is achieved with a relatively simple surgical procedure, such as skin grafting or suturing. All patients will be followed up until 6 weeks after application to observe adverse events related to the application of PRP and the dressings. This study was designed to address and compare the safety and efficacy of PRP covered with a hydrocolloid dressing versus a gelatin sheet. If successful, this combination therapy may be an alternative to bioengineered skin substitutes containing living cells and lead to substantial progress in the management of chronic skin ulcers.

Ethics and dissemination: The study protocol was approved by the Institutional Review Board of Kansai Medical University (KMU Number 0649-1, 4 August 2014: V.1.0). The findings of this trial will be disseminated through peer-reviewed journals, and national and international scientific meetings as well as to the patients.

Trial registration number: UMIN000015689.

\section{INTRODUCTION}

Chronic skin ulcers caused by diabetes mellitus, venous insufficiency, pressure sores,

\section{Strengths and limitations of this study}

- The current protocol will provide evidence to compare the efficacy of platelet-rich plasma (PRP) covered with a hydrocolloid dressing and that of PRP covered with a gelatin sheet, in the treatment of chronic skin ulcers.

- This study is a historical control study.

- Only subgroups are randomised, and the small number of study participants may affect generalisability.

collagen disease, trauma or radiation are intractable and costly problems in healthcare. ${ }^{1-3}$ In particular, diabetic foot ulcers and venous leg ulcers are frequent and costly complications of the underlying diseases. The prevalence of diabetic foot ulcers ranges from $4 \%$ to $10 \%$ among patients with diabetes, and the lifetime incidence is reported to be as high as $25 \% .{ }^{23}$ It has been reported that venous leg ulcers recur in $72 \%$ of cases, and the recurrence rate after skin grafting is $48 \%$ within 1 year. ${ }^{4}$ With the development of tissue engineering, bioengineered skin substitutes and genetically derived growth factors have progressed greatly in recent years with respect to the treatment of chronic skin ulcers. ${ }^{5-7}$ However, new treatment options for chronic skin ulcers are needed.

Platelet-rich plasma (PRP) is blood plasma enriched with platelets. Autologous PRP is a source of autologous growth factors, such as platelet-derived growth factor (PDGF), transforming growth factor- $\beta$ (TGF- $\beta$ ) and vascular endothelial growth factor (VEGF) ${ }^{89}$ PRP has been used clinically in a variety of treatments for bone defects, tendon injuries, cartilage injuries, cosmetic surgeries and chronic ulcers. ${ }^{10}$ Regarding the efficacy of PRP in the treatment of chronic wounds, PRP has, in 
many reports, been described as improving the rate of healing compared with standard care. ${ }^{11} 12$ On the other hand, in other studies, no major differences have been found in the healing outcomes of leg ulcers between PRP treatment groups and control groups. ${ }^{11} 12$

A drug delivery system (DDS) is a type of technology for enhancing the biological activity of growth factors with a very short in vivo half-life after application. Biodegradable hydrogels of gelatin with an isoelectric point (IEP) of 5.0 have been designed and developed for the sustained release of positively charged growth factors, such as basic fibroblast growth factor (bFGF), PDGF-BB and TGF- $\beta 1 .^{13-15}$ The efficacy of a sustained release system for epidermal growth factor was also recently reported. ${ }^{16}$ PDGF-BB and TGF- $\beta$ are major growth factors involved in PRP for improving the wound healing process, and enhanced angiogenesis by gelatin microspheres impregnated with PRP has been reported. ${ }^{13}$ Additionally, gelatin hydrogel sheets have been used to achieve the sustained release of growth factors and can be applied on wounds directly as a wound dressing. ${ }^{17}$ The wound healing effect of PRP covered with this sheet is expected to be enhanced by the sustained release of growth factors from the gelatin sheet as a result of its biodegradation after application.

In this report, we designed an exploratory clinical study to investigate the safety and efficacy of PRP application covered with our gelatin sheet compared with PRP covered with a hydrocolloid dressing, in the treatment of chronic skin ulcers. In addition, we estimated the contents of PDGF-BB, TGF- $\beta 1$ and VEGF in PRP, and compared these values and the degree of wound healing associated with the gelatin sheet.

\section{MATERIALS AND ANALYSIS}

\section{Primary objective}

The objective of this study is to evaluate the safety and efficacy of the administration of PRP in the treatment of chronic skin ulcers that are not expected to heal using conventional treatments.

The safety and efficacy of PRP covered with a gelatin sheet versus a hydrocolloid dressing will also be evaluated.

\section{Methods and design}

Open-label, non-randomised, controlled clinical trial.

\section{Design}

This study is a prospective cohort study using historical data as a control. A group treated with PRP application covered with a gelatin sheet and a group treated with PRP application covered with a hydrocolloid dressing have been set. Patients will be randomised to the gelatin sheet subgroup or the hydrocolloid dressing subgroup, and a randomisation-based comparison between these groups is expected to achieve significant improvements in accuracy with a lack of bias. This comparison will provide useful information for designing and conducting future trials.

\section{Setting and participants}

This study is being conducted at Kansai Medical University Hirakata Hospital and Kansai Medical University Takii Hospital. Patients with chronic skin ulcers are being referred by physicians and identified through a number of wound care clinics in Osaka Prefecture and surrounding prefectures.

\section{Inclusion criteria}

1. Patients 20 years of age or older with informed consent.

2. The presence of the following chronic skin ulcers:

- Not healing for at least 1 month with conventional treatments;

- Not expected to respond to conventional treatments;

- Expected to be closed with autologous skin grafting or suturing after PRP treatment;

- An ulcer area between 3 and $30 \mathrm{~cm}^{2}$ (that can be applied with autologous PRP).

3. Written informed consent.

\section{Exclusion criteria}

1. Patients showing unstable haemodynamics due to ischaemic heart disease or impaired blood coagulation, as in clotting disorders.

2. Patients taking anticoagulants or antithrombotics judged by the investigator or subinvestigator to be inappropriate as participants since the drugs may affect the clotting system.

3. Any of the following systemic diseases:

- Uncontrolled diabetes mellitus (defined as a glycated haemoglobin level of $\geq 10 \%$ according to the latest laboratory data obtained within 28 days before registration);

- Requiring the continued use of oral corticosteroid therapy (>20 mg/day prednisolone equivalent);

- A history of malignant tumours with a disease-free interval of 3 years or less.

4. Patients who are or plan to be pregnant.

5. Other patients judged by the investigator or subinvestigator to be inappropriate as participants of this study.

\section{Analysis of subgroups}

In this study, patients will be randomised to either the gelatin sheet subgroup or hydrocolloid dressing subgroup at a ratio of 1:1 without stratification. Randomisation will be performed by assigning random numbers from random number tables to the treatment conditions.

\section{Interventions}

\section{Preparation of PRP}

PRP is prepared using the Magellan Autologous Platelet Separator System (Medtronic Inc, Minneapolis, Minnesota, 
USA) and its basic disposable kit. PRP is prepared by sampling blood using $60 \mathrm{~mL}$ syringes of the basic disposable kit filled with an anticoagulant, and separating and concentrating platelets to threefold or more compared with that observed in the peripheral blood using the Magellan Autologous Platelet Separator System. The obtained PRP is activated as described in our previous study. ${ }^{8}{ }^{9}$ Briefly, a 1:1 (v/v) mixture of $0.5 \mathrm{M} \mathrm{CaCl}_{2}$ (Otsuka Pharmaceutical Factory, Inc, Tokushima, Japan) and autologous thrombin is prepared in advance as an activator. A 10:1 (v/v) mixture of PRP and the activator is incubated for $5 \mathrm{~min}$ at room temperature. Platelets of prepared PRP are counted, and the concentrations of growth factors (TGF- $\beta 1$, PDGF-BB and VEGF) in activated PRP are also quantified. If platelets cannot be separated and concentrated threefold or more compared with that in the peripheral blood, the study will be discontinued.

\section{Preparation of the gelatin sheet}

Acidic gelatin with an IEP of 5.0 (Nitta Gelatin, Osaka, Japan) is to be used in this study. Gelatin hydrogel sheets of $12 \times 3 \mathrm{~cm}$ in size are prepared at the Department of Biomaterial, Field of Tissue Engineering, Institute for Frontier Medical Sciences, Kyoto University. ${ }^{16}$ Briefly, $40 \mathrm{~g}$ of aqueous gelatin solution (5 wt\%) preheated at $40^{\circ} \mathrm{C}$ is mixed with $80 \mu \mathrm{L}$ of aqueous glutaraldehyde (GA, Wako Pure Chemical Industries, Osaka, Japan) solution (25 wt \%). The resulting mixture is poured into polystyrene dishes and left at $4^{\circ} \mathrm{C}$ for $12 \mathrm{~h}$ for chemical cross-linking of gelatin. Then, the formed hydrogel sheets are placed in $100 \mathrm{mM}$ glycine aqueous solution, followed by agitation for $1 \mathrm{~h}$ at room temperature to inactivate the residual unreacted aldehyde groups of GA. The gelatine hydrogel sheet is washed with double-distilled water, freeze-dried and sterilised with ethylene oxide gas. To remove the remaining ethylene oxide gas, the resulting hydrogel sheet is kept at $40^{\circ} \mathrm{C}$ for 3 days under vacuum conditions.

\section{Application of activated PRP and dressing changes}

After debridement of the chronic skin ulcers, activated PRP is applied to the lesions. Following the administration of PRP, the wound is dressed with a gelatin hydrogel sheet or conventional hydrocolloid dressing (DuoActive; Convatec Japan, Tokyo, Japan). The wound is occluded with the above dressing material for 5 days from the beginning of the administration of PRP and treated thereafter by applying ointment, such as Azunol ointment (Nippon Shinyaku Co Ltd, Kyoto, Japan) or wound dressings, until 6 weeks after the administration of PRP.

\section{Subsequent therapy}

The use of bFGF will be prohibited starting 3 or more days prior to the administration of PRP (wash-out period of bFGF) and during the study period. Negative-pressure wound therapy must also not be performed during the study period. After completion of the study period
(6 weeks after PRP administration or the day on which wound closure is judged to be possible), no particular restrictions will be imposed. Concomitant therapies, such as a compression treatment, in cases of venous leg ulcers, will be continued and applied before entry, because it is important to evaluate the efficacy of PRP under the same conditions.

\section{Digital photography for the healing assessment}

Using a digital camera, digital images of the wounds will be taken with a calibrator (CASMATCH; BEAR Medic Corp, Tokyo, Japan) placed on the skin adjacent to the wound. The colour and size of the images can be adjusted using the CASMATCH and an image editing software program (Adobe Photoshop; Adobe Systems Inc, California, USA) to assess the wound and granulation areas as well as the wound bed. As with the primary endpoint, the wound evaluation committee members will assess the extent of wound closure.

\section{Primary endpoint}

The primary endpoint is the 'time to wound closure'.

Time to wound closure: The time (number of days) from the administration of PRP to wound closure, spontaneous wound closure or wound closure with a simple surgical procedure. The results of the evaluation will be confirmed by the wound evaluation committee members consisting of independent physicians separate from the physicians involved in the study to ensure the neutrality, objectivity and reproducibility of the evaluations.

The evaluation criteria for the possibility of wound closure during the investigation are as follows:

1. Judged to be closed by spontaneous healing.

2. Judged to be closed using a simple surgical procedure with an ulcer area less than $3 \mathrm{~cm}^{2}$ (when two or more of the four conditions below are fulfilled):

- Seventy-five per cent or more of the wound area is covered by granulation tissue.

- Bones, tendons and underlying tissues are covered with granulation tissue.

- Local infection is controlled clinically (no adverse events due to infection).

- The wound depth measured by using a depth gauge is reduced by an average of $50 \%$ or more.

\section{Secondary endpoints}

- Percentage of wound reduction: Before and after the application of PRP, the wound is photographed once a week at dressing change, and the wound area is measured serially.

- Degree of improvement in the blood flow: The skin perfusion pressure or ankle brachial index is measured at dressing changes before and 1 and 4 weeks after the application of PRP.

- Correlations between the 'time to wound closure' and the platelet count or growth factor levels in the PRP area. 


\section{Blinding}

In this study, a data monitoring committee was not organised, although the wound closure will be independently measured under blinding by three specialists. The patients will be unblinded, and unblinded investigators will apply PRP and the change dressings.

\section{Sample size}

This study is a prospective cohort study using historical data as controls, with a total of 30 patients for registration, a feasible size for a pilot study.

\section{Study schedule}

The schedule of the study assessments and evaluations is shown in table 1 . The study period will be from the day of PRP application to 6 weeks after PRP application. Data for evaluating the efficacy and safety of this study will be collected at enrolment, baseline, day 5 and weeks $1,2,3,4,5$ and 6 after application or the day of wound closure.

\section{Statistical analysis}

The statistical analysis will be performed on the following variables:

- Time to wound closure;

- Percentage of wound reduction;

- Improvement in the blood flow;

- Correlations of the 'time to wound closure' with the platelet count and/or growth factor levels in the PRP area;

- Adverse events related to the application of PRP.

The efficacy will be evaluated according to the time from PRP application to wound closure. The survival curves in an existing control group based on historical data and the PRP-treated group until wound closure will be evaluated according to the Kaplan-Meier method, and a stratified analysis will be performed using the log-rank test. A stratified analysis of the number and percentage of patients who show wound closure will be performed using Fisher's exact test (95\% CI).

In addition, the optimal dose of PRP for wound healing will be estimated from the platelet count and growth factor concentrations in the PRP area.

The incidences of adverse events, and adverse events that can be causally related to PRP application, will be evaluated based on the event and severity. An interim analyses and auditing will not be planned; however, the investigators will monitor adverse events and other unintended effects of the trial interventions.

\section{ETHICS}

This study is being conducted in compliance with the ICH-GCP and in agreement with the latest revision of the Declaration of Helsinki, Pharmaceutical Affairs Law, and all applicable Japanese laws and regulations, as well as any local laws and regulations, and all applicable guidelines.






\section{Participant consent}

Informed consent will be obtained from all potential study participants using the Institutional Review Board-approved informed consent form. The clinical investigator informs the potential study participant of all pertinent aspects of the study, and the participant must sufficiently understand the content of the information form before providing written consent. The consent form must be dated and signed by both the investigator and participant. The participants are also informed that their medical care will not be affected if they do not choose to participate in this study. The consent forms will be retained at Kansai Medical University Hirakata Hospital and Kansai Medical University Takii Hospital, and the information form and a copy of the consent form will be handed to the participant. Whenever the investigator obtains information that may affect the participant's willingness to continue participation in the study, the investigator or subinvestigator will immediately inform the participant and record this observation and subsequently reconfirm the participant's willingness to continue participation in the study.

\section{DISSEMINATION}

The findings of this trial will be disseminated through peer-reviewed journals, and national and international scientific meetings as well as to the patients.

\section{DISCUSSION}

This study was designed to address the safety and efficacy of PRP treatment for chronic skin ulcers using a gelatin hydrogel sheet that can sustain the levels of growth factors in the PRP material. This study will be the first controlled trial to evaluate the efficacy of PRP covered with a hydrocolloid dressing and PRP covered with a gelatin sheet in the treatment of chronic skin ulcers. The efficacy of PRP for the treatment of chronic wounds in clinical trials is still controversial. ${ }^{10-12}$ Our gelatin hydrogel sheet has been applied in clinical trials mainly as a DDS for bFGF, and its efficacy and safety have been reported. ${ }^{17}{ }^{18}$ bFGF can be used clinically in Japan; however, the application of bFGF is not approved in other countries. ${ }^{71920}$ The clinical usage of growth factors in these countries is limited. PRP can be prepared from the patient's blood or donated platelets in all countries. Some bioengineered skin substitutes that provide growth factors secreted by living cells have been reported to be effective for chronic skin ulcers, although they are costly and access is also limited to only a few areas. ${ }^{21-23}$ Our gelatin sheet is freeze-dried and can be stored at room temperature for months. Therefore, it is possible to use this combination therapy consisting of PRP and a gelatin sheet anywhere when needed, and its efficacy might be superior to that of bioengineered skin substitutes, as these treatments have a similar mechanism of action in secreting growth factors in a sustained manner. Regarding the limitation of this study, this study included ulcers of various causes that could be potential for bias by different concomitant therapies. Therefore, we will plan the clinical study limited to one ulcer cause with standardised concomitant therapy in the next step. This is our first trial using the PRP/gelatin hydrogel sheet and we focused mainly on safety in this study, and evaluate the efficacy in the next step limited to one ulcer cause.

If successful, this intervention may lead to substantial and important changes in the management of increasingly prevalent chronic skin ulcers, such as diabetes ulcers and venous leg ulcers.

Contributors NM, NK and KK initiated the study design. NM prepared the first draft of the manuscript. NM, NK, TO, TH, KS and KK will treat patients and conduct the clinical trial. NM will generate the allocation sequence, enrol participants and assign the participants to the interventions. MM, MY and YT provided contributions from the engineering aspect for the gelatin sheets, and prepared and supplied the sheets. NM is a grant holder. All authors contributed to refining the study protocol and approved the final manuscript.

Funding This work was supported by the Japan Society for the Promotion of Science (JSPS) KAKENHI grant number 24390399 (https://kaken.nii.ac.jp/d/p/ 24390399.en.html).

Competing interests None declared.

Patient consent Obtained.

Ethics approval Institutional Review Board of Kansai Medical University.

Provenance and peer review Not commissioned; externally peer reviewed.

Open Access This is an Open Access article distributed in accordance with the Creative Commons Attribution Non Commercial (CC BY-NC 4.0) license, which permits others to distribute, remix, adapt, build upon this work noncommercially, and license their derivative works on different terms, provided the original work is properly cited and the use is non-commercial. See: http:// creativecommons.org/licenses/by-nc/4.0/

\section{REFERENCES}

1. Vuorisalo S, Venermo M, Lepäntalo M. Treatment of diabetic foot ulcers. J Cardiovasc Surg (Torino) 2009;50:275-91.

2. Singh N, Armstrong DG, Lipsky BA. Preventing foot ulcers in patients with diabetes. JAMA 2005;293:217-28.

3. Wu SC, Driver VR, Wrobel JS, et al. Foot ulcers in the diabetic patient, prevention and treatment. Vasc Health Risk Manag 2007;3:65-76

4. Morimoto N, Takemoto S, Kawai K, et al. Immediate evaluation of neovascularization in a grafted bilayered artificial dermis using laser Doppler imaging. Ann Plast Surg 2014;72:84-8.

5. Ehrenreich M, Ruszczak Z. Update on tissue-engineered biological dressings. Tissue Eng 2006;12:2407-24.

6. Mason C, Manzotti E. Regenerative medicine cell therapies: numbers of units manufactured and patients treated between 1988 and 2010. Regen Med 2010;5:307-13.

7. Uchi $\mathrm{H}$, Igarashi $\mathrm{A}$, Urabe $\mathrm{K}$, et al. Clinical efficacy of basic fibroblast growth factor (bFGF) for diabetic ulcer. Eur J Dermatol 2009;19:461-8.

8. Kakudo N, Minakata T, Mitsui T, et al. Proliferation-promoting effect of platelet-rich plasma on human adipose-derived stem cells and human dermal fibroblasts. Plast Reconstr Surg 2008;122:1352-60.

9. Kushida S, Kakudo N, Morimoto N, et al. Platelet and growth factor concentrations in activated platelet-rich plasma: a comparison of seven commercial separation systems. J Artif Organs 2014;17:186-92.

10. Sommeling CE, Heyneman A, Hoeksema $\mathrm{H}$, et al. The use of platelet-rich plasma in plastic surgery: a systematic review. J Plast Reconstr Aesthet Surg 2013;66:301-11.

11. Saad Setta $H$, Elshahat A, Elsherbiny $K$, et al. Platelet-rich plasma versus platelet-poor plasma in the management of chronic diabetic foot ulcers: a comparative study. Int Wound J 2011;8:307-12. 
12. Martinez-Zapata MJ, Martí-Carvajal AJ, Solà I, et al. Autologous platelet-rich plasma for treating chronic wounds. Cochrane Database Syst Rev 2012;10:CD006899.

13. Matsui M, Tabata $\mathrm{Y}$. Enhanced angiogenesis by multiple release of platelet-rich plasma contents and basic fibroblast growth factor from gelatin hydrogels. Acta Biomater 2012;8:1792-801.

14. Ishida K, Kuroda R, Miwa M, et al. The regenerative effects of platelet-rich plasma on meniscal cells in vitro and its in vivo application with biodegradable gelatin hydrogel. Tissue Eng 2007;13:1103-12.

15. Tabata Y. Nanomaterials of drug delivery systems for tissue regeneration. Methods Mol Biol 2005;300:81-100.

16. Doerler M, Eming S, Dissemond J, et al. A novel epidermal growth factor-containing wound dressing for the treatment of hard-to-heal venous leg ulcers. Adv Skin Wound Care 2014;27:456-60.

17. Miyoshi M, Kawazoe T, Igawa $\mathrm{HH}$, et al. Effects of bFGF incorporated into a gelatin sheet on wound healing. J Biomater Sci Polym Ed 2005;16:893-907.
18. Marui A, Tabata $\mathrm{Y}, \mathrm{Kojima} \mathrm{S}$, et al. A novel approach to therapeutic angiogenesis for patients with critical limb ischemia by sustained release of basic fibroblast growth factor using biodegradable gelatin hydrogel: an initial report of the phase I-lla study. Circ $J$ 2007:71:1181-6.

19. Morimoto N, Yoshimura K, Niimi M, et al. Novel collagen/gelatin scaffold with sustained release of basic fibroblast growth factor: clinical trial for chronic skin ulcers. Tissue Eng Part A 2013;19:1931-40.

20. Takemoto S, Morimoto N, Kimura Y, et al. Preparation of collagen/ gelatin sponge scaffold for sustained release of bFGF. Tissue Eng Part A 2008;4:1629-38.

21. Langer A, Rogowski W. Systematic review of economic evaluations of human cell-derived wound care products for the treatment of venous leg and diabetic foot ulcers. BMC Health Serv Res 2009;9:115.

22. Yannas IV, Orgill DP, Burke JF. Template for skin regeneration Plast Reconstr Surg 2011;127(Suppl 1):60S-70S.

23. Priya $S G$, Jungvid $H$, Kumar $A$. Skin tissue engineering for tissue repair and regeneration. Tissue Eng Part B Rev 2008;14:105-18. 